\title{
Gynecological Surgery and Machine Learning: Complications and Length of Stay Prediction
}

\author{
Oleg METSKER ${ }^{\mathrm{a}}$, Georgy KOPANITSA ${ }^{\mathrm{b}, 1}$, Anton MALUSHKO ${ }^{\mathrm{a}}$, Eduard \\ KOMLICHENKO $^{\mathrm{a}}$, Katerina BOLGOVA ${ }^{\mathrm{b}}$ and David PASKOSHEV ${ }^{\mathrm{b}}$ \\ ${ }^{a}$ Almazov National Medical Research Centre, Saint-Petersburg, Russia \\ bITMO University, Saint-Petersburg, Russia
}

\begin{abstract}
In this study we are developing predictive models for a length of stay after a gynecological surgery, complications and the length of the surgery using machine learning methods. The study was performed with the data of patients with the diseases of the female reproductive system. The patients were admitted to the Almazov National Medical Research Centre (Saint-Petersburg, Russia) within the period 2010-2020. The study included 8170 electronic medical records of inpatient episodes including 3500 operation protocols. The data included anamnesis of life, anamnesis of disease, laboratory tests, severity, outcome of a surgery, main and comorbid diagnosis, complications, case outcome. The dataset was randomly split into $70 \%$ train and $30 \%$ test datasets. Validation with the test dataset provided the following prediction metrics for the length of stay after a surgery model. Training score: AUC of ROC: 0.9582230976834093 ; K-fold CV average score: -8.73; MSE: 5.65; RMSE: 2.83 .
\end{abstract}

Keywords. Surgery, complications, duration, machine learning, length of stay, prediction

\section{Introduction}

Gynecology embraces a group of diseases that originate in the female reproductive organs. Extensive gynecologic surgery often entails meticulous dissection near the bladder, rectum, ureters, and great vessels of the pelvis [1]. Complications of gynecologic surgery include hemorrhage, infection, thromboembolism, and visceral damage [2]. The risk of complications depends on the extent and approach to surgery and patient characteristics. The common complications of a surgery relate to injuries of viscera and occur during extensive resections for the treatment of cancer or when anatomy is distorted due to infection or endometriosis [3]. So, prediction of complications and the duration of stay in the hospital will allow a better and more personalized treatment and more efficient planning of hospital resources particularly in the situation of resource shortage especially in the developing countries. Data-driven machine learning methods have shown evidences of efficiency in health care and in the risk prediction of gynecological surgeries in particular $[4,5]$.

${ }^{1}$ Corresponding Author, Georgy Kopanitsa, ITMO University, Saint-Petersburg, Russia; E-mail: georgy.kopanitsa@gmail.com. 
For example, the study [6] developed a prediction model for a 30-day morbidity after gynecological malignancy surgery. With a bootstrap-corrected concordance, index of the nomogram incorporating these three predictors was 0.656 (95\% CI, 0.608-0.723).

A Prediction Model and Risk Calculator for Surgical Mortality and Serious Morbidity After Primary Cytoreductive Surgery [7] included 6 preoperative clinical variables: age, chronic hypertension requiring medication, ascites, white blood-cell count, hematocrit, and serum creatinine. The model AUC was 0.73.

In this study we are developing predictive models for a length of stay after a gynecological surgery, complications and the length of the surgery using machine learning methods.

\section{Methods}

The study was performed with the data of the patients with the diseases of the female reproductive system of the Almazov National Medical Research Centre, SaintPetersburg, Russia. Participation criteria were: female gender, and the following diagnoses:

- $\quad$ N39.4 Other specified urinary incontinence

- N84.0 polyp of corpus uteri

- D25.1 Intramural leiomyoma of uterus

- N83.2 corpus albicans cyst

- D27 Benign neoplasm of ovary

- $\quad$ I10 Essential (primary) hypertension

- D25.0 Submucous leiomyoma of uterus

- D25.2 Subserosal leiomyoma of uterus

- $\quad$ N85.0 Endometrial hyperplasia

- N80.1 Endometriosis of ovary

- N84 Polyp of female genital tract

- C54.1 Malignant neoplasm of endometrium

- N80.0 Endometriosis of uterus

- N80.3 Endometriosis of pelvic peritoneum

- N81.1 Cystocele

- N81.2 Incomplete uterovaginal prolapse

- N81.3 Complete uterovaginal prolapse

- $\quad$ N81.8 Other female genital prolapse

- $\quad$ N97.1 Female infertility of tubal origin

- $\quad$ N97.8 Female infertility of other origin

- D50.0 Iron deficiency anemia secondary to blood loss (chronic)

- $\quad$ N93 Other abnormal uterine and vaginal bleeding

- N73.6 Female pelvic peritoneal adhesions (postinfective)

- O00.1 Tubal pregnancy

- C56 Malignant neoplasm of ovary

- N99.3 Prolapse of vaginal vault after hysterectomy

- N92.0 Excessive and frequent menstruation with regular cycle

- D28.2 Benign neoplasm of uterine tubes and ligaments

- $\quad$ E11 Type 2 diabetes mellitus 
- Q51.3 Bicornate uterus

- D25.9 Leiomyoma of uterus, unspecified

- N70.1 Chronic salpingitis and oophoritis

- $\quad$ N99.4 Postprocedural pelvic peritoneal adhesions

- D50.9 Iron deficiency anemia, unspecified

- Q51 Congenital malformations of uterus and cervix

- N75.0 Cyst of Bartholin's gland

- N92.1 Excessive and frequent menstruation with irregular cycle

- Q51.8 Other congenital malformations of uterus and cervix

- N70 Salpingitis and oophoritis

- $\quad$ N90.6 Hypertrophy of vulva

- N39 Other disorders of urinary system

- Q52 Other congenital malformations of female genitalia

- D39.1 Neoplasm of uncertain behavior of ovary

- D28 Benign neoplasm of other and unspecified female genital organs

- O02 Other abnormal products of conception

- C53 Malignant neoplasm of cervix uteri

- N88 Other noninflammatory disorders of cervix uteri

- C54 Malignant neoplasm of corpus uteri

The patients were admitted to the Almazov National Medical Research Centre within the period 2010-2020. The study included 3745 electronic medical records of inpatient episodes including operation protocols. The electronic medical records included the following data: anamnesis of life, anamnesis of disease, laboratory tests, type, severity, outcome of surgery, main and comorbid diagnosis, complications, case outcome. The dataset was randomly split into $70 \%$ train and $30 \%$ test datasets.

\subsection{Predictive modelling}

To find the most efficient model we made a grid search experiment. The dataset was split into training (70\% random selection) and testing (30\% random selection). Each experiment ran in the setting of stratified 5-fold cross-validation i.e., random $80 \%$ of training dataset was used for training and random $20 \%$ of training dataset for testing. Target class ratios in the folds were preserved. Mean Absolute Error (MAE) was used as a performance metric. All the measurements were performed separately per dataset and per model parameter to determine the best parameters for classifiers as well as optimal data preprocessing. After determining the optimal dataset and model parameters, we performed a validation with the testing dataset. We used a series of classification models available within scikit-learn pool: Random Forest, Gradient Boost and Voiting regressors for the selection of the best predictive methods to be applied within the proposed scheme. The Shapley index was calculated to identify most common predictors for the complications.

\section{Results}

The top performing model for the duration of stay is an XGBR regressor with the following parameters: XGBRegressor(base_score=None, booster=None, 
colsample_bylevel=None, colsample_bynode $=$ None, colsample_bytree $=$ None, gamma=None, interaction_constraints $=$ None, max_depth=None, monotone_constraints $=$ None, gpu_id=None, importance_type='gain', num_parallel_tree $=$ None, random_state $=$ None, reg_alpha $=$ None, reg_lambda $=$ None, scale_pos_weight $=$ None, $\quad$ subsample $=$ None, $\quad$ tree_method $=$ None, validate parameters $=$ False, verbosity $=0$ ). Validation with the test dataset provided the following prediction metrics for the length of stay after a surgery model (Figure 1): Training score: AUC of ROC: 0.9582230976834093; K-fold CV average score: -8.73; MSE: 5.65;RMSE: 2.83 .

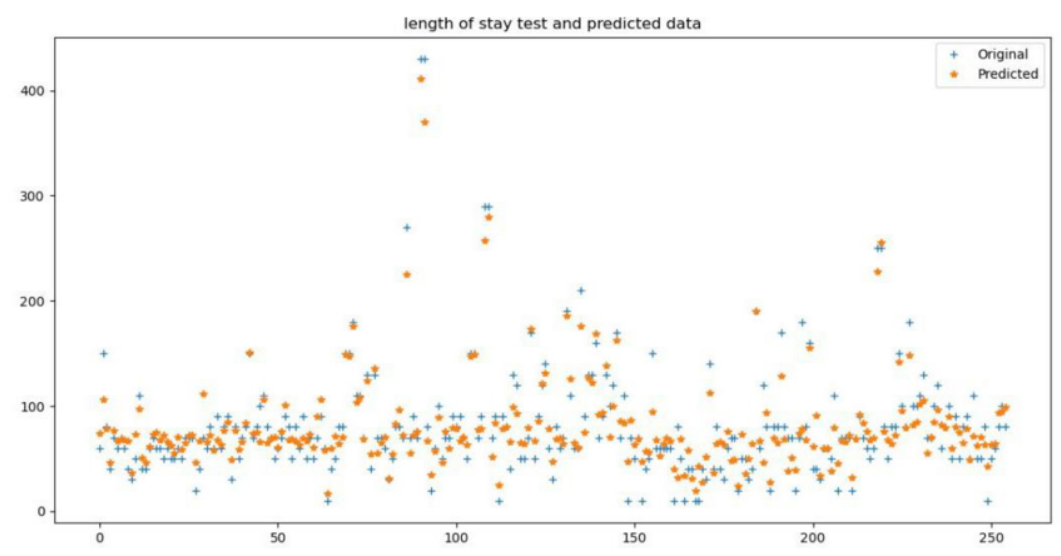

Figure 1. Length of a stay in hospital model performance in hours.

Figure 2 presents the Shapley values for the prediction of the thrombosis complication after a surgery. The predictive model has shown the following evaluation metrics' values: AUC of ROC test score: 97.0; K-fold CV average score: 0.96.

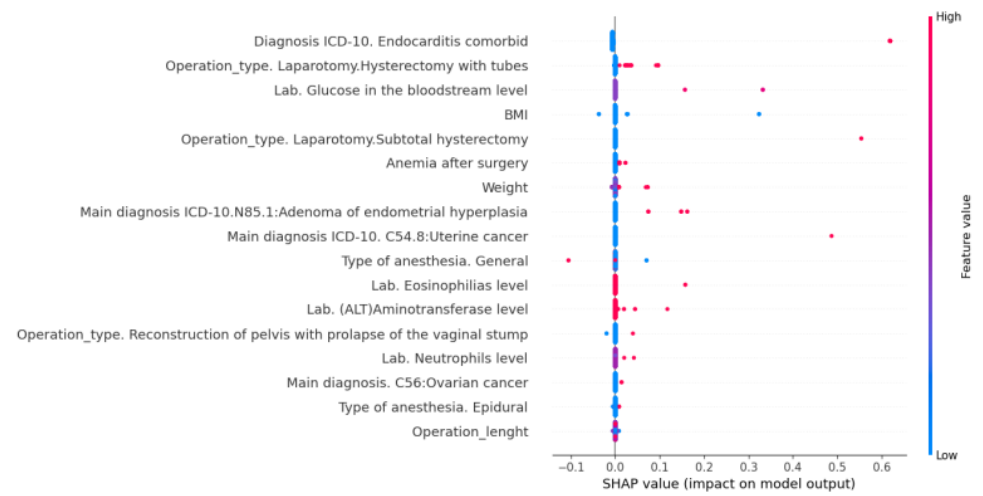

Figure 2. Shapley index for the thrombosis prediction

Validation with a test data set provided the following metrics for the duration of a surgery model (Figure 3): Test score AUC of ROC: 0.9663954919164502 ; K-fold CV average score: -3.32 , MSE: 350.67 , RMSE: 175.34 


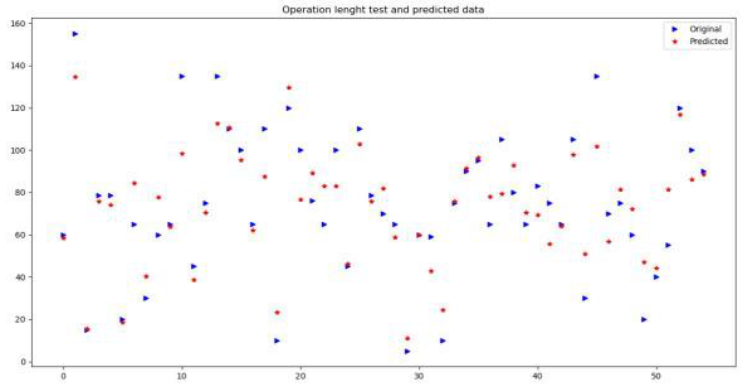

Figure 3. Length of a surgery

\section{Discussion and conclusion}

This study presents implementation of predictive models for the duration of stay after a surgery, complications and duration of a surgery. The models provide a higher precision than the state-of-the-art models [4-7]. Identification of risk factors using the results of the features importance analysis can support clinicians in the early analysis of complications and planning the preventive measures. Prediction of the duration of stay can support in planning and allocating resources in the situation of the resource shortage.

\section{Acknowledgement}

This work was supported by the Russian Foundation for Basic Research, grant number 20-37-70047. The work of Georgy Kopanitsa was financially supported by the Government of the Russian Federation through the ITMO fellowship program.

\section{References}

[1] Bashan-Gilzenrat KA. Surgical Critical Care and Emergency Surgery: Clinical Questions and Answers: Second Edition; 2018;210:431-437.

[2] Kalogera E, Bakkum-Gamez JN, Jankowski CJ, Trabuco E, Lovely JK, Dhanorker S, Grubbs PL, Weaver AL, Haas LR, Borah BJ, Bursiek AA, Walsh MT, Cliby WA, Dowdy SC. Enhanced recovery in gynecologic surgery. Obstet. Gynecol. 2013;12(2).

[3] Latif N, Kobie J, Mitra N, Burger R, Morgan M, Guintoli R, Ko E. A Prediction Model and Risk Calculator for Surgical Mortality and Serious Morbidity After Primary Cytoreductive Surgery. J. Gynecol. Surg. 2018;34: 1-5.

[4] Tsui KL, Chen N, Zhou Q, Hai Y, Wang W. Prognostics and health management: A review on data driven approaches. Math. Probl. Eng. 2015;2015:793161.

[5] Metsker O, Kopanitsa G, Bolgova E. Prediction of childbirth mortality using machine learning. Studies in Health Technology and Informatics. 2020;273:109-114.

[6] Shim SH, Lee SJ, Dong M, Suh JH, Kim SY, Lee JH, Kim SN, Kang SB, Kim J. Prediction model for 30-day morbidity after gynecological malignancy surgery. PLoS One. 2017 Jun 1;12(6):e0178610.

[7] Cham S, Chen L, St Clair CM, Hou JY, Tergas AI, Melamed A, Ananth CV, Neugut AI, Hershman DL, Wright JD. Development and validation of a risk-calculator for adverse perioperative outcomes for women with ovarian cancer. Am J Obstet Gynecol. 2019 Jun;220(6):571.e1-571.e8. 